\title{
The Research on Repeater Coordination Based on Band Plan
}

\author{
Peng $\mathrm{Xu}^{1} \&$ Jian-Yu Zheng ${ }^{2}$ \\ ${ }^{1}$ Electrical and Information School, Jinan University, China \\ ${ }^{2}$ School of Translation Studies, Jinan University, China \\ Correspondence:Peng Xu,Electrical and Information School, Jinan University, Zhuhai 519070, China. E-mail: \\ 1364969501@qq.com
}

Received: April 12, 2014

Accepted: March 8, 2015

Online Published: July 25, 2015

doi:10.5539/cis.v8n3p145

URL: http://dx.doi.org/10.5539/cis.v8n3p145

\begin{abstract}
It is very troublesome that repeaters could interfere with one another. So we need to coordinate them. In our paper, we flexibly use the technology CTCSS (Continuous Tone-coded Squelch System) to avoid interfere and accommodate more simultaneous users with the least repeaters. Then we propose a mixed-frequency-band repeater network consist of some single-frequency-point repeater networks. Secondly, considered that the causes and solutions of interference might occur in a repeater network, we propose a "band plan" to reduce the "multi-path" interference in frequency and phase. Then combined with the most efficient repeater networks, we apply our model about "band plan" into the plat area. According to some limiting conditions, we bring the reality datum into our repeater network model to accommodate 1,000 simultaneous users, and conclude that the minimum of repeaters is 76 . Finally, we apply our plan into a mountain area, in order to meet some new circumstances there, we adjust our"band plan" and repeaters to avoid interfere.
\end{abstract}

Keywords: repeater networks, band plan, optimization algorithm, "multi-path" interference, repeater coordination in mountain areas

\section{Introduction}

The Very High Frequency radio spectrum involves line-of-sight transmission and reception (Repeater Map Locator). An ingenious equipment can overcome this sight-to-light limitation — repeaters, which pick up signals, amplify them, and retransmit them at a different frequency. However, repeaters can be interfered with other repeaters unless far enough apart or transmit at sufficiently separated frequencies. Fortunately, the technology CTCSS (Continuous Tone-coded Squelch System), nicknamed "private line"(PL), can be used to solve interference problems (International Telecommunication Union.Amateur and Amateur-satellite service). Therefore, how to use this technology to coordinate repeaters is very vital.

Xieping J. analyzed the source of interfere in repeater networks. After doing some computational work, he proposed solutions to eliminate same frequency interference in a repeater network (Xieping J., 2015). Ping S. et al. explored measures about improving the reliability of repeater stations (Ping S., 2010). In addition, as to repeater coordination, there are numerous correlational research done by some researchers. Ziwei M. analyzed relevant factors which could affect repeater coordination, which makes this paper consider into more influence factors (Ziwei M., 2011). Shuhong W. established a mathematical model about multi-path fading, which provides theoretical basis for solving the problems about multi-path fading (Shuhong W., 2010). Jianzhong S. proposed the CTCSS technology in order to solve interfere problem about communication between repeaters (Jianzhong S.).

Considering those methods above, they are unsuitable to cope with current situations about repeater coordination. Therefore, combined with the advantages of those methods, we propose "band plan" and design an exquisite repeater network to avoid interfere among repeaters. Meanwhile, we apply our methods into plat areas and mountain areas. We also adjust our initial plans many times to adapt to those new circumstances in those areas.

\section{Model for a Repeater Network in a Mountain Area}

The interfere among repeaters has been troublesome for users. With the help of technology CTCSS, this phenomenon has improved greatly. But in real life, we want to use the least repeaters to make simultaneous users communicate. Therefore, through flexible use the technology CTCSS to avoid interfere, we propose a 
mixed-frequency-band repeater network to achieve this goal. Then we propose a "band plan" to reduce the "multi-path" interference in frequency and phase. After proposing these methods, we apply them into the plat areas and serve the most simultaneous users. Finally, we also coordinate repeaters in a mountain area by improving our initial plan.

\subsection{Model for Eliminating Interfere in Repeater Networks}

\subsubsection{The Theory of CTCSS Technology}

Though repeaters can improve VHF radio's disadvantages, different repeaters will interfere with each other, just like Figure 1

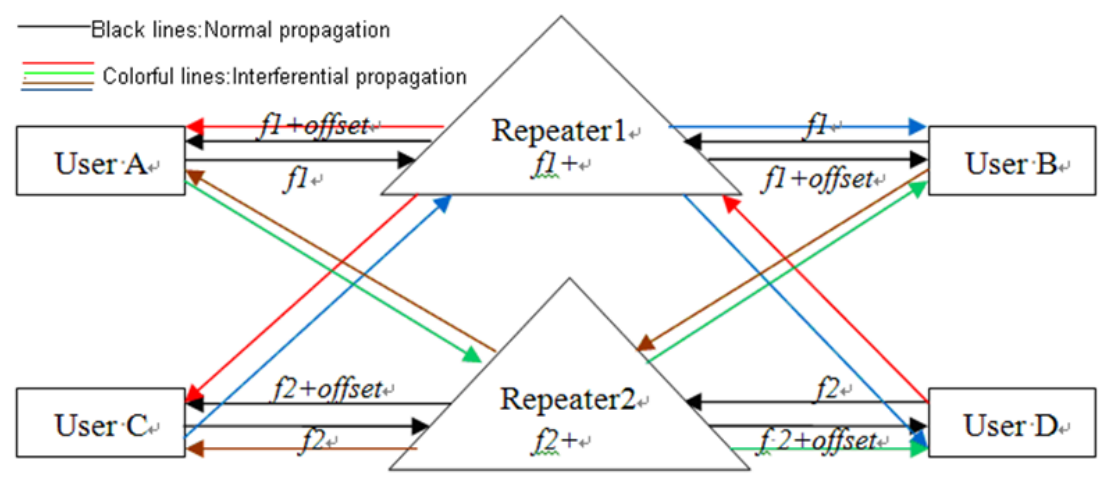

Figure 1. the Interfere from Different Repeaters

When repeaters are too close with each other or transmit at similar frequencies, interfere will happen. Just like Figure1 shown ,the black lines mean the normal transmission mode by a repeater, while other colorful lines mean the interfere between the two repeaters.

In order to avoid that, the technology CTCSS, nicknamed "private line"(PL), is put forward. A repeater's transmission frequency or receive frequency is not a fixed frequency but a changeable frequency so that it forms a frequency band. Then frequencies for CTCSS tone encoding systems are to be per EIA standards. Level is to be adjusted to produce the maximum transmitter deviation $+/-600 \mathrm{~Hz}$ or minimum transmitter deviation $+/-400$ $\mathrm{Hz}$ (The Metropolitan Coordination Association.Coordination Guidelines). That is why CTCSS technology can be used in repeaters, which can absolutely receive signals with $+/-400 \mathrm{~Hz}$ even $+/-600 \mathrm{~Hz}$ offsets from the center frequency. Besides, when more than one users are on the same channel (co-channel users), CTCSS can filter out the other users if they are using a different CTCSS tone or they have not a CTCSS tone.

Besides, we need know how to flexibly use the CTCSS technology. Just like Figure 2 shown, when users want to communicate with each other through a repeater with the technology PL, they need set their transceivers to a special PL tone mode. However, if there is another co-channel user transmitting a signal with the same PL tone to the repeater, the original user will get interfered. Besides, the signal will not be transmitted unless it uses the right PL tone. So it is ideal to set two co-channels to accommodate these user groups in an area, so that they can communicate without any interference. And the technology CTCSS achieves that goal.

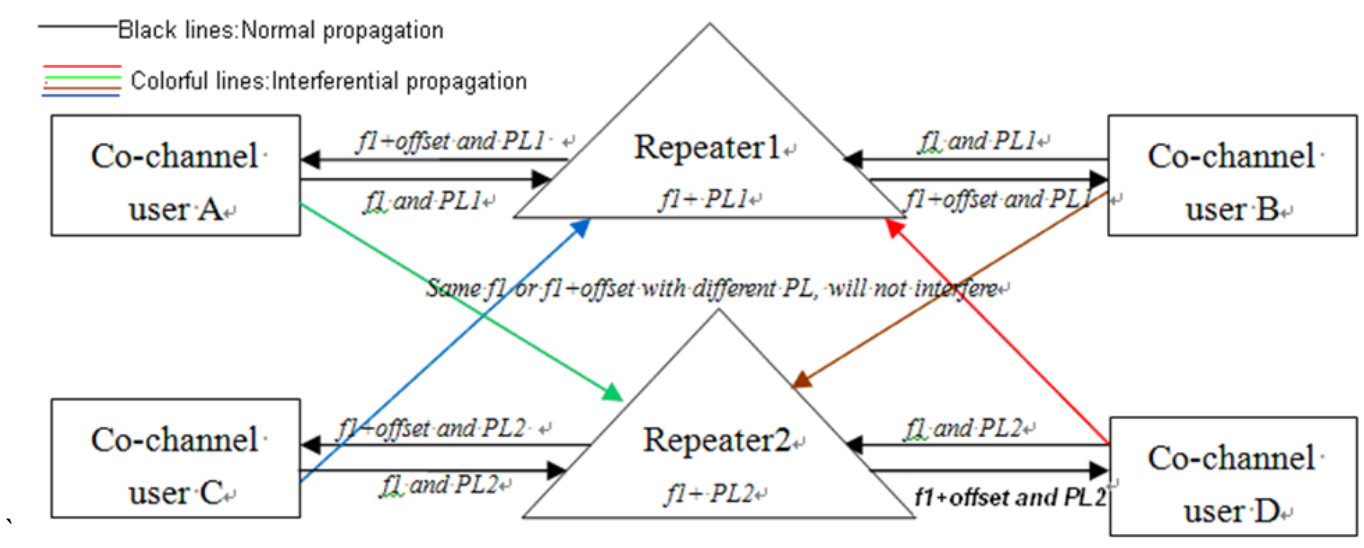

Figure 2.the Two Co-channels by Using the CTCSS Technology 
However, CTCSS technology also causes many problems. Just like Figure 3 shown, in order to serve more users at a certain frequency, more repeaters with a particular PL have to be set up. For example, if there are 54 user groups need be served at the same frequency with different PL tones, there has to be 54 different PL tones available and 54 repeaters needed.

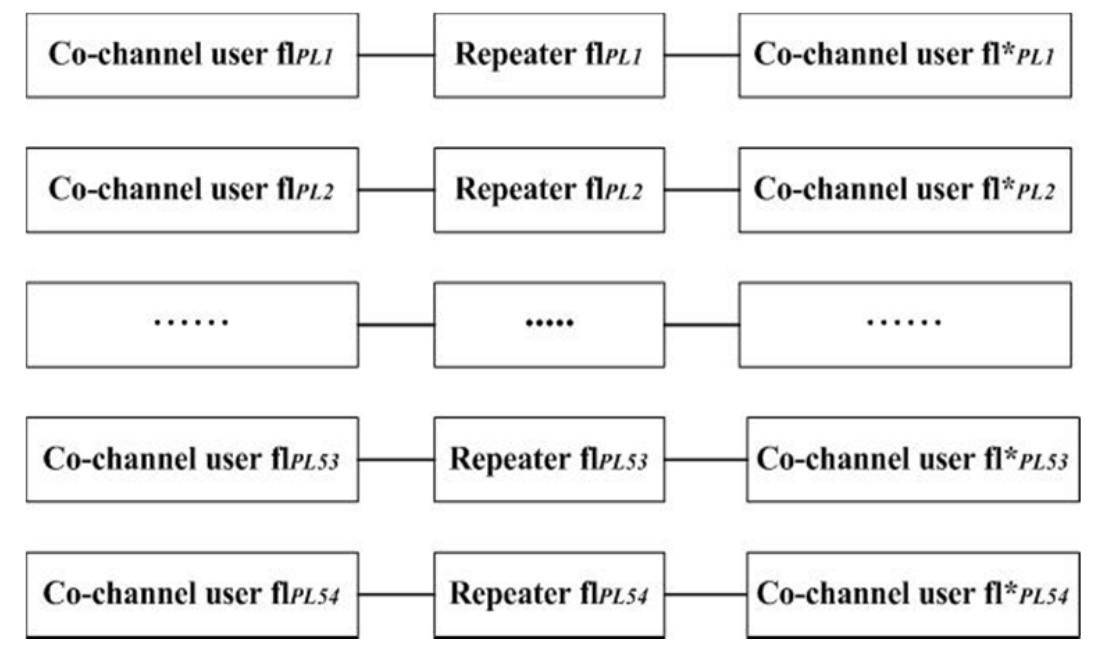

Figure 3. PL-PL-PL network mode

As a matter of fact, a signal with a particular PL tone can be transmitted through a repeater with the same PL tone or without any PL tones. With the aim of minimizing the numbers of repeaters and providing network service to more users, it's an optimal way to let users communicate with each other through a non-PL repeater and use a particular PL on their transceivers. As a result, the original repeater network can be replaced by a public network with a non-PL repeater, which greatly reduces the number of repeaters. The network mode is just like Figure 4 shown.

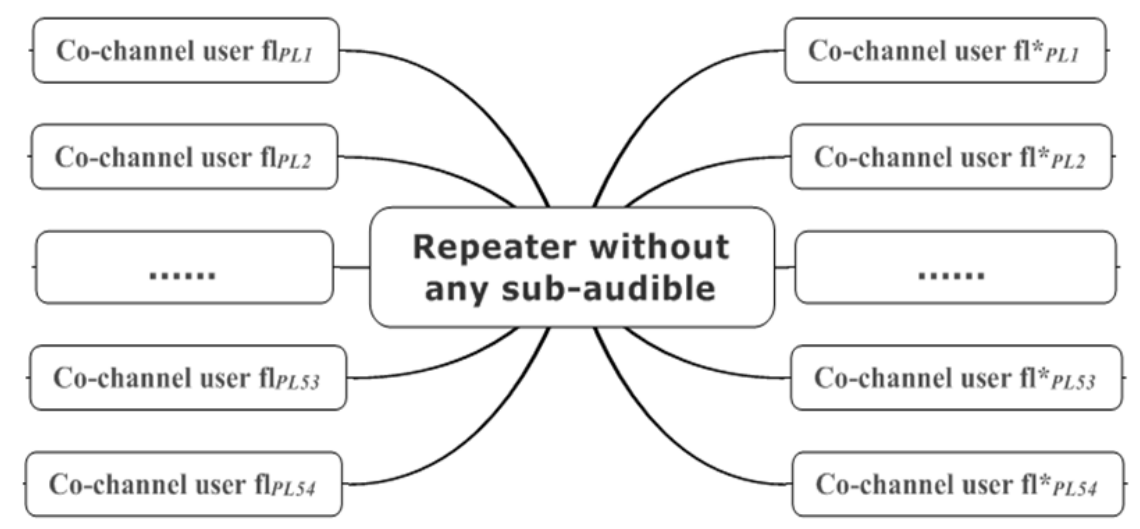

Figure 4. PL-No PL-PL network mode

\subsubsection{The Causes and Solutions of Interference in a Repeater Network}

Before designing a repeater network, we have to know about the causes and solutions about interference.

(1)The geographical interference

This phenomenon is caused because two repeaters are too close between each other.There are three methods to solve the problem: Make two repeaters far enough apart; Make two repeaters nearby transmit at sufficiently separated frequencies; Using CTCSS technology.

\section{(2)Self-interference}

When a repeater gets a radio, if it is larger than the isolation between transmitting and receiving antennas, the feedback signal from the transmit antenna into the receive antenna will cause the repeater to go into feedback 
oscillation (Lee M., 2008). Therefore, self-interference is caused. To solve this phenomenon, we can assume that repeater's isolator is well performed enough to prevent this kind of interference in this paper.

\section{(3)Loop interference}

We can take an example to illustrate this kind of interfere. When the transmission frequency of a repeater is the receiving frequency of B repeater, and B repeater's transmission frequency is exactly A repeater's receiving frequency, then the signal is doing an infinite loop between the two repeaters. In order to solve this problem, we can only use the repeaters whose receiving frequencies are lower than their transmission frequencies. In this way, this kind of interference will no longer exist in our repeater network.

(4)Users interference:

With the same PL, two users use the same repeater to transmit signals at the same frequency. At that time, users interference often happens. As to this kind of interfere, there are so many agreements to avoid it, such as: wait 5 seconds before starting the communication to determine whether there is someone else to use the same channel; the time out principle: the repeater network will cut off the line when someone's communication time is more than 3 minutes.

\section{(5)"multi-path effect" interference}

When a signal can be transmitted into another point in a repeater network by many different paths, it could cause multi-path effect. Besides, multi-path effect in a repeater network can be divided into two cases:"multi-path effect" interference in frequency and "multi-path effect" interference in phase

Firstly, we will take an example to illustrate"multi-path effect" interference in frequency. Just like Figure 5, when a user $\mathrm{D}$ is listening music from a FM radio at $146.2 \mathrm{MHz}$ frequency, and in the same area, user B is chatting on the repeater network at $145.6 \mathrm{MHz}$ frequency with his friend $\mathrm{A}$. We can see it clearly from Figure 5, a signal from user A can be transmitted to user B and user D's area by two way: repeater1-repeater3 or repeater1-repeater2-repeater3. So when the signal from user A is transmitted at $145 \mathrm{MHz}$ frequency by the second way, it will reach to user B's area at $146.2 \mathrm{MHz}$. As a result, user D listening to music from FM radio at 146.2MHz frequency will hear user A's signals at the same time. So it is impossible for user D to appreciate the music any more.

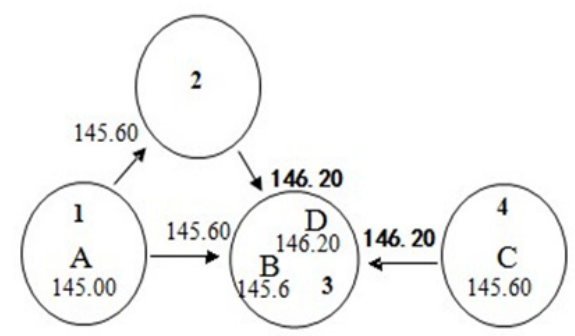

Figure 5. The Process of "Multi-Path Effect" Interference

Besides, if a signal can be transmitted into any places covered by this network by many different ways, though the receiver could accept the signal from the sender, it will take different times to transmit the signal by different paths because of the delay of different repeaters. This phenomenon could make the received signals interfere with each other, so that it affects the user's influence. And we call this phenomenon "multi-path effect" interference in phase.

All in all, as to the complicated questions above, it is necessary to design a very delicate repeater network to avoid "multi-path" interference. It is also the emphasis we deal with in this paper.

\subsubsection{Model for Non-Interference "Band Plan" in Repeater Networks}

With the help of technology CTCSS, we hope more users can use VHF radio to communicate. So we need to build a mixed-frequency-band repeater network consist of some single-frequency-point repeater networks to achieve this goal. As to this mixed-frequency-band repeater network, it is very vital to propose a reasonable "band plan" to coordinate repeaters. However, before designing this network, we need introduce some concepts about a mixed-frequency-band repeater network and single-frequency-point repeater network. We can distinguish them from the perspective of frequency band.

A single-frequency-point repeater network only serves at a certain frequency. When a signal is transmitted through a repeater, all the other repeaters in this network will also be activated and transmit the same signal. 
While a mixed-frequency-band repeater network serves at the multiple frequencies. It's exactly a combination of some single-frequency-point repeater networks,which means there will be frequency overlapped among each single-frequency-point repeater networks .And Figure 6 shows frequency overlaps, where different color lines could be overlapped.

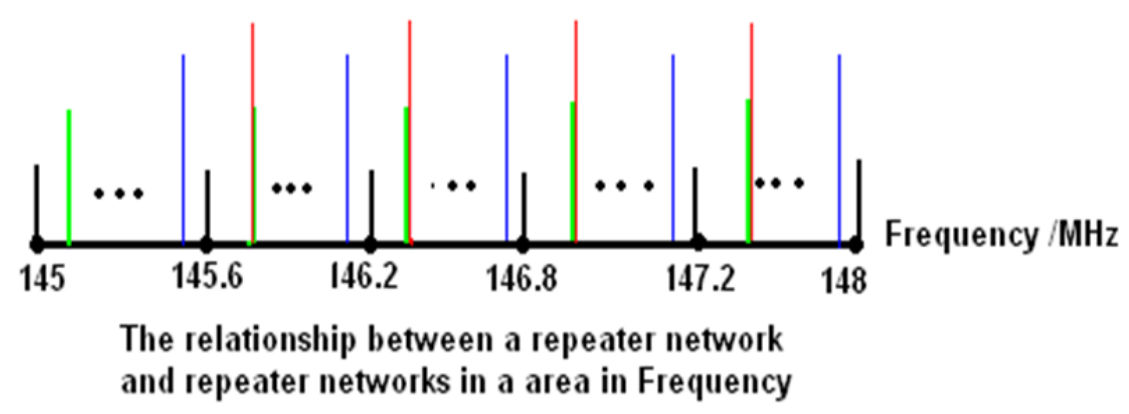

Figure 6. Frequency Overlaps in a Repeater Networks

As to achieve the repeater coordination in a mixed-frequency-band repeater network, we have to minimize interference among these stations and maximize use ratio of the limited radio spectrum according to the specific frequency or frequencies in a specific area. Besides, we have to obey the following principles to propose a "band plan":

(1)The new repeater station should avoid any kinds of interference with the existing stations;

(2)We can know all the parameters about the repeaters in a repeater network into which we want put a new repeater in order to obtain frequencies for a new repeater.

(3)When using a radio spectrum band in an area, it is necessary to make the repeater 's receiving frequency lower than the transmission frequency;

(4)From 144 to $146 \mathrm{MHz}$, the channel spacing is $20 \mathrm{KHz}$. While from 146 to $148 \mathrm{MHz}$, the channel spacing is $15 \mathrm{KHz}$.

Then we can propose a free-interference "band plan" for a mixed-frequency-band repeater network. In order to show our "band plan" clearly, we can set some conditions to follow about repeater coordinators:

(1)The spectrum available is 145 to $148 \mathrm{MHz}$;

(2)we need to design a repeater network for a circular flat area whose radius is 40 miles with the minimum repeaters.

(3)The transmitter frequency in a repeater network is either $600 \mathrm{kHz}$ above or $600 \mathrm{kHz}$ below the receiver frequency.

(4)There are 54 different PL tones available

Base on those conditions, the biggest problem facing to us is to prevent all kinds of interference in a mixed-frequency-band repeater network :

(1)In order to avoid the geographical interference,we will obey the requirement about the channel spacing

(2)In order to avoid the self-interference, we assume that the technology has developed enough .

(3)In order to avoid the loop interference, we can use the repeater whose receiving frequency lower than the transmission frequency.

(4)In order to avoid users interference, we use common agreements above to reduce it.

(5)In order to avoid the "multi-path" interference. If we do forbid any the transmission frequency pairs of repeaters being the same with another repeater covered with the same repeater networks in an area, it will not happen.

When all interference problems are solved, we can propose a model for non-interference "band plan" in a repeater network based on the spectrum band from $145 \mathrm{MHz}$ to $148 \mathrm{MHz}$. And our "band plan" is shown as Table 1. 
Table 1. Model for non-interference "band plan" in a repeater network

\begin{tabular}{cccccc}
\hline \multicolumn{6}{c}{ Repeateri1Repeateri2Repeateri3Repeateri4Repeateri5 } \\
\cline { 3 - 6 } 1 & $(\mathrm{MHz})$ & $(\mathrm{MHz})$ & $(\mathrm{MHz})$ & $(\mathrm{MHz})$ & $(\mathrm{MHz})$ \\
\cline { 2 - 6 } & 145.00 & 145.60 & 146.20 & 146.80 & 147.40 \\
2 & 145.02 & 145.62 & 146.22 & 146.82 & \\
3 & 145.04 & 145.64 & 146.24 & 146.84 & \\
4 & 145.06 & 145.66 & 146.26 & 146.86 & \\
5 & 145.08 & 145.68 & 146.28 & 146.88 & \\
6 & 145.10 & 145.70 & 146.30 & 146.90 & \\
$\vdots$ & $\vdots$ & $\vdots$ & $\vdots$ & $\vdots$ & \\
30 & 145.58 & 146.18 & 146.78 & 147.38 & \\
\hline
\end{tabular}

\subsection{A Repeater Network in a Flat Area}

In order to preferably show our model for non-interference "band plan" in a mixed-frequency-band repeater network in a flat area,we need set the limiting conditions:minimizing the number of repeaters to cover the whole area;accommodating 1000 simultaneous users, 40-mile covering radius; all users in the area can use a certain network to communicate; 54 different PLs.

\subsubsection{The Determination of the Coverage Radius of Repeaters}

According to the relevant parameters about repeaters in America, we analyzed them carefully and found out that repeaters with 30-miles coverage radius was the most popular. So in our model for non-interference "band plan" in a repeater network in a flat area, the coverage radius of repeaters is 30 miles.

\subsubsection{The Determination About the Structure of a Repeater Network}

Based on the conditions above, in order to cover the whole area with the minimum of repeaters, we propose following rules to determine the locations of repeaters and the coverage pattern:

Firstly,we set a structure matrix $\mathrm{A}(80,80)$ to represent a flat area whose radius is 40 -mile. In this matrix, every elements represents the corresponding geographical location. And their structures all include these properties:

- En indicates whether there exists a repeater, When " $E n$ is 1 " indicates there is a repeater, otherwise, there is no repeaters.

- $\operatorname{Inpf}$ represents input frequency of a repeater

- Outf is the output frequency of a repeater

- $\quad P L$ is the type of $P L$

Therefore, there is

$$
\mathrm{A}(80,80)=\text { struct('En',[ ],'Inpf',[ ],'Outf',[ ],'PL',[ ]); }
$$

And there is one thing to note, because the minimum spacing of co-channel repeaters is 70 miles (The Metropolitan Coordination Association.Coordination Guidelines), unless they don't use different PLs, it is impossible to coexist two repeaters at the same frequency in the same area.

To achieve this goal, the simulation has to follow these rules:

- The minimum separation distance to another repeater with the first adjacent channel is 25 miles

- Minimize the number of the repeaters

- Cover the whole area

Based on each units of the matrix, the simulation will proceed as follows:

(1) Determine a matrix $(80,80)$ to delegate the flat area is $\mathrm{A}(\mathrm{i}, \mathrm{j})$ 
(2) Randomly generate the locations of repeaters by setting EN to 1

(3) Determine the first unit as the basis

(4) Search out the adjacent units through the distances of each center

(5) Delete the nearest unit

(6)Check whether the whole area is covered, Yes - go to (7); No - recover the latest deleted unit and exit.

(7) Determine the farthest unit as a basis and go to (4)

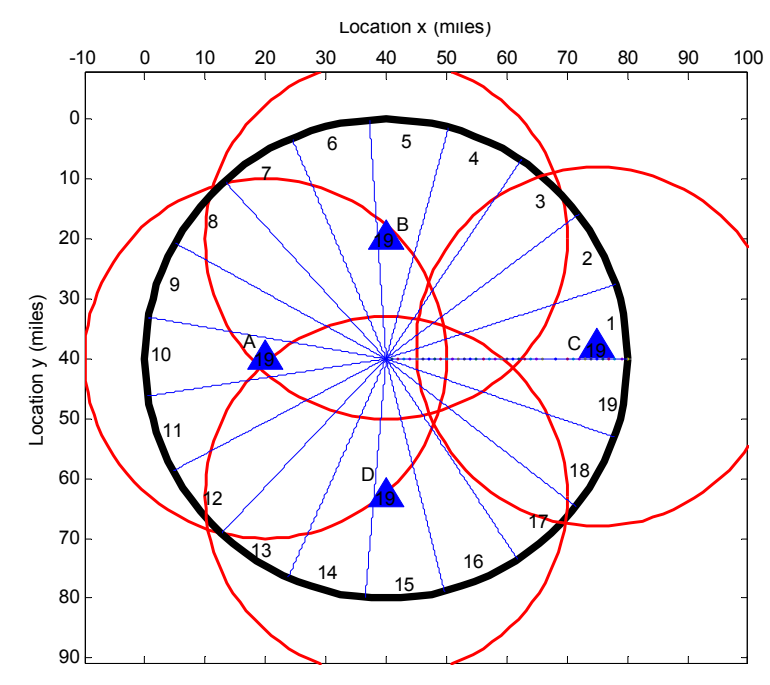

Figure 7. The locations of a repeater group in a repeater network

From the Figure 7, we can see the red circle as the most basic network. If there are 4 repeaters whose coverage radium is 30 miles located like that, the whole area can be covered. Therefore, a signal can be transmitted from the initial repeater to any places in the area through the other repeaters. Besides, repeaters in every network can be built at point A, B, C and D. All the four repeaters can constitute a repeater network in the area.

\subsubsection{The Establishment About an Efficient Repeater Network}

Through the last part, we know there need at least 4 repeaters in a network. We need to determine the relationship between a single-frequency-point repeater network and a mixed-frequency-band repeater network by band plan. According to Figure 8 ,we researched the band plan below, and found that all networks in this area showed a new relationship. That's a combination of a unique network. Hence, we only need to study a kind of frequency in the area.

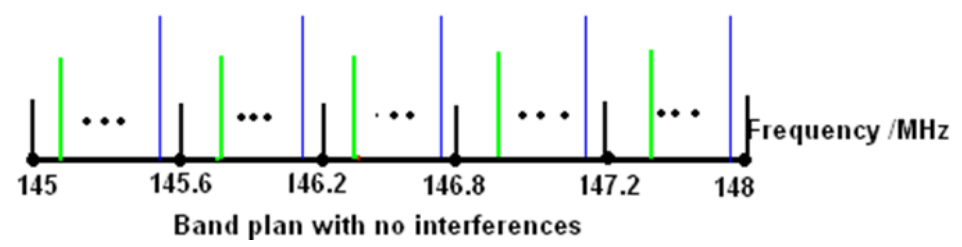

Figure 8. Band plan with no interference

Now we begin to set up an efficient mixed-frequency-band repeater network, which can serve at least 1000 users at the same time. Because the radius in the area is 40 miles and there are 54 different PLs, we have to set up $1000 / 54 \approx 19$ repeater networks. Because there need be 4 repeaters in every repeater network according the conclusion above. Therefore, we need $19 \times 4=76$ repeaters to cover the whole area. It is the 76 repeaters that can permit at least 1000 users to communicate with others in the area whose radius is 40 miles by using 76 different PLs. Therefore, the 76 repeaters also constitute a mixed-frequency-band repeater network.

\subsection{A Repeater Network in a Mountain Area}


According to the conditions which are the same with the last part 2.2, now we need to design a model for non-interference "band plan" in mountain area based on the previous repeater network in a flat area.

\subsubsection{Assumptions}

Designing a repeater network in a mountain area is a very complicated problem. Therefore, we need set some reasonable assumptions to guarantee us to build a perfect network:

(1)Considered that the geographical features of mountain area is very complicated,so we only discuss the mountains' heights in the area.

(2)After doing researches about mountainous areas, if we only research the height relations among three mountains, we find that there are three modes about that being worthy of study: two higher mountains are on the either sides and the shorter one is in the middle; two shorter mountains are on the either sides and the higher one is in the middle; three mountains' heights decrease in line.

(3)Assuming that a mountain can represent a region, where signals can be transmitted at a specific frequency.

(4)Assuming that the delay about a repeater is so short that it will not cause "multi-path effect" interference in phase.

(5)Assuming that no matter where the highest mountain is in this mountain area, the repeater on it can cover the whole area whose radius is 40 miles.

\subsubsection{Design Principles About "Band Plan" in Mountain Area}

Considered that "multi-path" interference in frequency and phase is very complicated, we can solve this problem step by step in the progress of designing the repeater network for this area.At the same time, we have to put forward these design principles:

(1)It is necessary to design a mixed-frequency-band repeater network to cover all the mountain areas.That is to say, any users can contact the others through at least one way. However, it is worth mentioning that there are some blind areas for the signals which are transmitted through the highest mountain.So those signals need be transmitted through the other repeaters to the blind areas.

(2)As to all kinds of interfere, we can use the same way used in the flat area to solve the problems about geographical interference, self-interference, loop interference and user interference.

(3)However,because it is very complicated to solve the problems about "multi-path" interference in frequency and phase, we need to design a new mixed-frequency-band repeater network.

\subsubsection{An Initial Repeater Networks}

(1)"band plan" for a mountain area

Firstly, we need avoid the multi-path interference in the mountain area. So just like the measures taken in the flat area,we can select $20 \mathrm{KHz}$ as the minimum channel spacing and do the same jobs used in the flat area.

After that, we can know that every mountains (like in the flat area) only has its own transmission channels in this area. So each of them has a unique repeater network, and these networks together can cover all the whole area.

(2) A new repeater network in a mountain area

To build up this network, we need obey the following principles:

Firstly, we set a repeater on the highest mountain. So when a user transmits its unique transmission $\mathrm{fc} \mathrm{MHz}$ there, he can connect any places in this area except those blind areas. At the same time, other users on the top of any other mountains can receive the signal from the highest mountain.

Secondly, as to the blind area, we also build repeaters with $\mathrm{fc}+0.6 \mathrm{MHz}$ receiving frequency on the tops of all mountains except the highest mountain.

According to those principles, when a user transmit a signal at the fcMHz frequency through the repeater on the highest mountain, another user in the blind can receive the signal at the $\mathrm{fc}+1.2 \mathrm{MHz}$ frequency. However,there is a new problem coming. Those users in the non-blind area can receive signals from the highest mountain at two kinds of frequencies: $\mathrm{fc}+0.6 \mathrm{MHz}$ and $\mathrm{fc}+1.2 \mathrm{MHz}$, which causes "multi-path" interference in frequency. We will discuss this phenomenon below.

There are two users using the same repeater network in this mountain area but they are in different mountains.If we do not make a band plan in advance, which means the transmission frequencies may be overlapped. For 
example, just like Figure 9 shown, if there is a user transmitting a signal at the $145.2 \mathrm{MHz}$ frequency. In our initial repeater network, this signal will be transmitted by other repeaters at two different frequencies: $145.2+0.6 \mathrm{MHz}$ and $145.2+1.2 \mathrm{MHz}$. Meanwhile, if there is another user transmitting a signal at $145.8 \mathrm{MHz}$ frequency in the same repeater network, multi-path interference in frequency will happen.

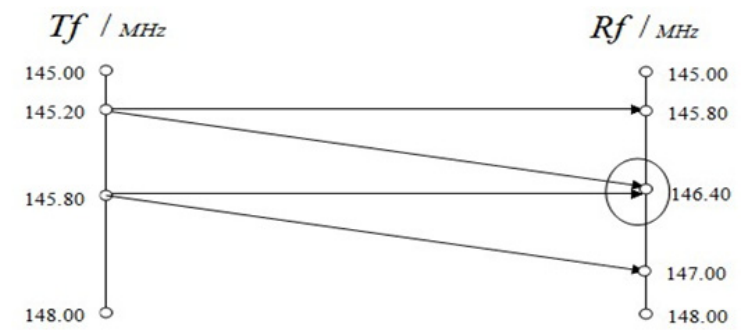

Figure 9. Multi-path interference at frequency in the mountain area

In order to solve this problem,we design a band plan for this repeater network in the mountain area. We can understand the method through Figure 10. Because our "band plan" only allows unique transmitting frequency in the network, so there is no overlap at the receiving frequencies.

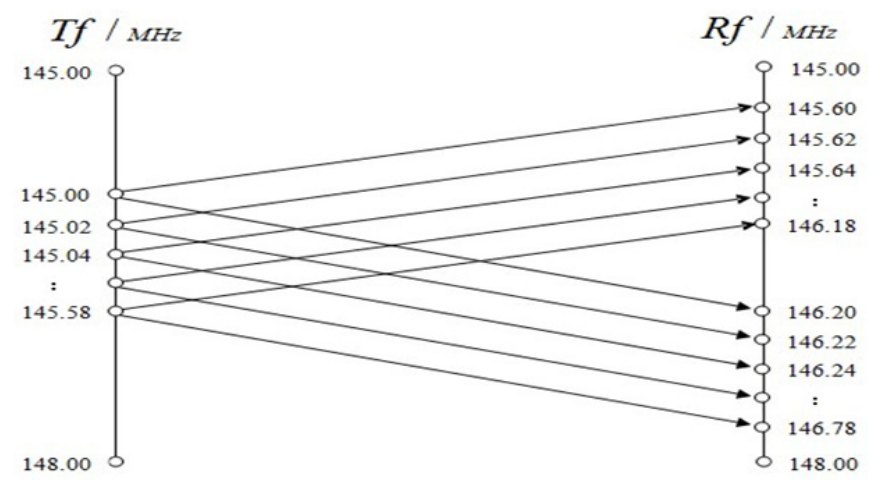

Figure 10. An non-interference band plan

\subsubsection{The test and Improvement About the Initial Repeater Network}

Now it occurs a new problem. Just like Figure 11 shown, when a user $\mathrm{y}$ in the mountain $\mathrm{G}$ tries to contact user $\mathrm{x}$ in the mountain D, they use our initial repeater network to contact. And there are two situations for user y to contact $\mathrm{x}$. The first one is that user $\mathrm{y}$ can contact user $\mathrm{x}$ through the path: $\mathrm{y}-\mathrm{E}-\mathrm{x}$ if the transmitter on the top of mountain $G$ can contact the receiver on the top of mountain $E$; the second one is that the transmitter has to contact the receiver on the highest mountain $\mathrm{C}$ if the transmitter on the top of mountain $\mathrm{G}$ can not contact the receiver on the top of mountain $\mathrm{E}$. But $\mathrm{x}$ is in the blind area of mountain $\mathrm{C}$, so the signal received by the mountain $\mathrm{C}$ has to contact the receiver on the mountain $\mathrm{E}$, and then transmits it into $\mathrm{x}$. Therefore, the path is $\mathrm{y}-\mathrm{G}-\mathrm{C}-\mathrm{E}-\mathrm{x}$. However, if a signal transmitted by user $\mathrm{y}$ reaches to user $\mathrm{x}$ through the two ways above, the frequencies are different.

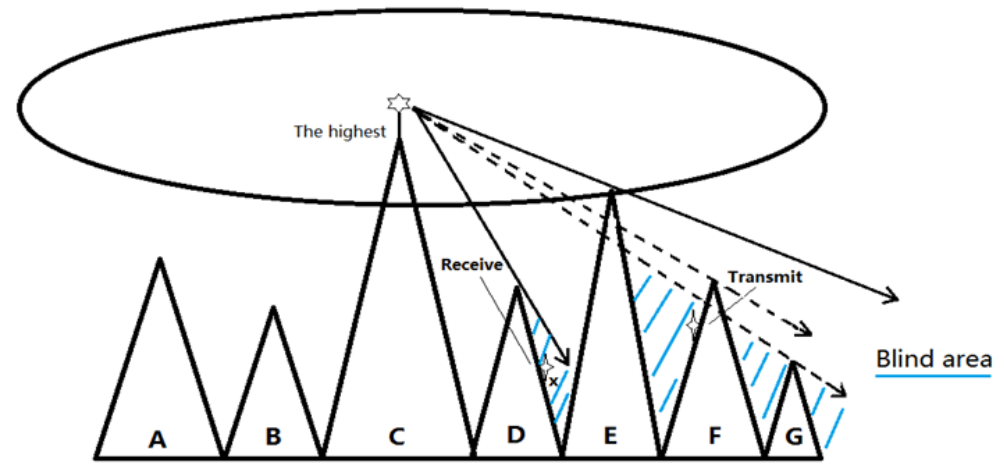

Figure 10. The working situation of repeaters in mountainous areas 
In order to solve the problem,we improve the initial repeater network according to the following principles:

(1)Every mountain only has a unique transmission frequency distributed by our band plan

(2)For every unique transmitting frequencies in different mountains, there is a unique repeater network covering the whole area;

(3)If a repeater on the highest mountain receives a frequency $f \mathrm{MHz}$ from the mountain area, it will transmit it at the $f-0.6 \mathrm{MHz}$ frequency.

(4)As to the other mountains, if a repeater on that mountain receives a $f \mathrm{MHz}$ signal, it will transmit it at the $f+0.6 \mathrm{MHz}$ frequency.

Therefore, the problem above has been solved by these principles. Even though a signal is transported through this two way, user $\mathrm{x}$ will receive signals at the same frequency.

\section{Conclusion}

Repeater coordination has been vital for users to communicate by VHF radio. In order to avoid all kinds of interfere, we put forward a model for eliminating interfere in repeater networks, so that every users could have a unique PL to communicate with the help technology CTCSS. Based on some limiting conditions, we applied our model for eliminating interfere in mixed-frequency-band repeater network into a plat area through designing a "band plan" in a repeater network. We find that there need to be at least 76 repeaters to accommodate 1,000 simultaneous users in the area whose radius is 40 miles. Then we adjust our method into a mountain area, we put forward a new "band plan"method for non-blind-area users to avoid the"multi-path" interference at frequency. Besides, we adjust some principles to let blind-area users receive a signal at the same frequency through different ways. Finally,we find our improvement about the initial repeater network in the mountain area achieve outstanding effects.

As to the future work, if there are more users wanting to use this network, it is necessary to create more repeaters and PLs. Besides, we need design more exquisite plans to avoid all kinds of interfere in the next step.

\section{References}

International Telecommunication Union.Amateur and Amateur-satellite service. Retrieved March 4, 2015, from http://life.itu.int/radioclub/ ars.htm

Jianzhong, S., Liren, Z., \& Da-peng, T. (2011). A Method to Optimally Allocate Repeaters. Digital Technology \& Application, 8, 21-21.

Lee, M., Keum, B., \& Lee, H, S., et al. (2008). A radio repeater interference cancellation model for mobile communication systems. Wireless and Mobile Communications, 376-381. http://dx.doi.org/10.1109/icwmc.2008.16

Ping, S. (2010) The Measure to improve the reliability of ultrashort wave repeater stations. Hydraulic Science and Technology, 1, 53-55.

Repeater Map Locator. Retrieved March 1, 2015, from http://www.usrepeaters.com/index

Shuhong, W. (2010). Mathematical Model and Analysis of Multipath Fading. Radio Engineering, 40(10),35-37.

The Metropolitan Coordination Association.Coordination Guidelines. Retrieved March 4, 2015, from http://www.metrocor.net/coordination_guidelines.trm\#WhyCoordinate

Xieping, J., \& Jiapin, X. (2015). Optimization of Co-channel Interference Based on Relay Cellular Network. Radio Engineering, 4.

Ziwei, M., Manqing, M., \& Ran, D. (2011). Impact Factor Analysis on Repeater Coordination. Proceedings of 2011 International Conference on Information Management and Engineering, 52(40). http://dx.doi.org/10.7763/IPCSIT.2012.V52.40

\section{Copyrights}

Copyright for this article is retained by the author(s), with first publication rights granted to the journal.

This is an open-access article distributed under the terms and conditions of the Creative Commons Attribution license (http://creativecommons.org/licenses/by/3.0/). 